\title{
Increased thalamo-cortical functional connectivity in patients with diabetic painful neuropathy: A resting-state functional MRI study
}

\author{
XIAOMEI LIU ${ }^{1 *}$, XIANGHONG XU ${ }^{1 *}$, CUNNAN MAO $^{1 *}$, PENG ZHANG $^{1}$, QING ZHANG $^{2}$, LANLAN JIANG $^{1}$,

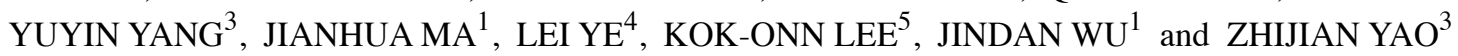 \\ ${ }^{1}$ Department of Endocrinology, Nanjing First Hospital, Nanjing Medical University, Nanjing, Jiangsu 210010; \\ ${ }^{2}$ Department of Endocrinology, The Affiliated Jiangning Hospital of Nanjing Medical University, Nanjing, \\ Jiangsu 210000; ${ }^{3}$ Department of Psychiatry, Nanjing Brain Hospital Affiliated to Nanjing Medical University, \\ Nanjing, Jiangsu 210029, P.R. China; ${ }^{4}$ National Heart Research Institute Singapore, National Heart Centre Singapore, \\ Singapore 169609; ${ }^{5}$ Department of Medicine, National University of Singapore, Singapore 119074, Singapore
}

Received May 17, 2020; Accepted February 10, 2021

DOI: $10.3892 /$ etm.2021.9940

\begin{abstract}
Functional changes in the brain of patients with painful diabetic neuropathy (PDN) have remained largely elusive. The aim of the present study was to explore changes in thalamo-cortical functional connectivity (FC) of patients with PDN using resting-state functional MRI. A total of 20 patients with type 2 diabetes mellitus (T2DM) with non-painful diabetic neuropathy (Group NDN), 19 patients with T2DM with PDN (Group-PDN) and 13 age-, sex- and education-matched healthy controls were recruited. The differences in thalamo-cortical FC among the three groups were compared. Patients in Group PDN had increased FC in the left thalamus, the right angular gyrus and the occipital gyrus as compared to those in Group NDN. Furthermore, patients in Group PDN had increased FC in the right thalamus and angular gyrus as compared to those in Group NDN. In conclusion, the present results suggested that the thalamo-cortical FC is increased in patients with T2DM and PDN. Furthermore, the increased FC in the thalamic-parietal-occipital connectivity may be a central pathophysiological mechanism for PDN. The
\end{abstract}

Correspondence to: Professor Jindan $\mathrm{Wu}$, Department of Endocrinology, Nanjing First Hospital, Nanjing Medical University, 68 Changle Road, Nanjing, Jiangsu 210010, P.R. China

E-mail:wujindandan@sina.com

Professor Zhijian Yao, Department of Psychiatry, Nanjing Brain Hospital Affiliated to Nanjing Medical University, 264 Guangzhou Road, Nanjing, Jiangsu 210029, P.R. China

E-mail: zjyao@njmu.edu.cn

*Contributed equally

Key words: diabetes mellitus, resting-state functional MRI, functional connectivity, thalamo-cortical, painful neuropathy, imaging study was retrospectively registered at ClinicalTrials.gov on 3 October 2018 (identifier no. NCT03700502).

\section{Introduction}

Among all patients with type 2 diabetes mellitus (T2DM), $\sim 60 \%$ have peripheral neuropathy and one-third of these have neuropathic pain (1). Chronic pain is the most common health problem in the developed world $(2,3)$. Diabetic neuropathy (PDN) is one of the most common cause of chronic pain (1). Furthermore, approximately two-thirds of patients with PDN also present with anxiety and depression (4), which contribute to a poor quality of life (5). However, the curative efficacy of PDN treatment remain unsatisfactory (1).

A previous study investigated the central pathophysiological mechanisms of PDN (5). The thalamus is the hub of the cortical-subcortical connections and is considered as the 'central core'. Furthermore, it is closely connected with regions involved in different emotional and cognitive tasks (6). The thalamus is also regarded as a relay station projecting to the cerebral cortex, which is responsible for sensations, such as pain (7). Patients with PDN have been indicated to exhibit a reduction in the volume of gray matter around the somatosensory cortex, which may have important implications for the long-term prognosis of DPN (8). A previous study also demonstrated that PDN was associated with thalamic and limbic dysfunctions, as well as impaired default and attention networks (5). Thus, the importance of the thalamus in PDN is being recognized.

Resting-state functional MRI (rs-fMRI) is a neuroimaging method that enables researchers to measure the activities of different brain regions (9). Furthermore, rs-fMRI has been used to explore the intrinsic functional connectivity (FC) of the brain in the resting state $(10,11)$. A previous study by our group indicated that patients with PDN had abnormal spontaneous activities in several brain regions, including somatosensory, cognitive and emotional activities, which were associated with increased insulin resistance, depression and anxiety (12). Furthermore, the duration of diabetes, glycated hemoglobin 
(HbA1c) levels, homeostasis model assessment-insulin resistance and the estimated glomerular filtration rate were significantly associated with abnormal spontaneous activity in the brain.

The present study aimed to explore abnormalities of the thalamo-cortical FC in patients with PDN using rs-fMRI. The study may provide novel information on the underlying mechanisms of PDN.

\section{Materials and methods}

Participants. A cross-sectional study was performed to explore the FC of the thalamus in patients with PDN using rs-fMRI at the Department of Endocrinology of Nanjing First Hospital (Nanjing, China) between September 2016 and March 2017. Patients were categorized into three groups: i) Patients with T2DM and PDN (Group PDN), ii) patients with T2DM and non-painful neuropathy (Group NDN) and iii) healthy subjects as a control (Group C). The protocol was approved by the Institutional Review Board of Nanjing First Hospital (Nanjing, China). All procedures were in accordance with the Declaration of Helsinki from 1964 and its later amendments or comparable ethical standards. Written informed consent was obtained from all participants.

The inclusion criteria for the patients were as follows: i) Voluntary participation and written informed consent; ii) age between 18 and 60 years with junior high school education or above; and iii) met the 1999 World Health Organization T2DM diagnostic criteria (fasting plasma glucose $\geq 7.0 \mathrm{mmol} / 1$ or 2-h postprandial glucose $\geq 11.1 \mathrm{mmol} / \mathrm{l}$ ) (13).

The criteria for the diagnosis of PDN were as follows: i) Neuropathy occurred after diagnosis of diabetes; ii) patients had clinical symptoms, such as pain, numbness or abnormal sensation; and iii) patients had abnormalities in one of the following five examinations: Ankle reflex, vibratory sensation, pressure sensation, temperature sensation and acupuncture pain (12).

The inclusion criteria for the healthy controls were as follows: i) Voluntary participation and written informed consent; ii) age between 18 to 60 years with junior high school education or above; iii) no history of diabetes and HbAlc levels of 4-6\%; and iv) normal results of anxiety and depression scales.

The exclusion criteria for all subjects were as follows: i) Left-hand writers; ii) neuropathy caused by other causes, such as cervical spondylosis, cerebral infarction, Green Barre syndrome, severe arteriovenous disease, drug neurotoxicity and renal insufficiency; iii) patients with severe cerebral vascular disease; iv) disorders such as depression, anxiety or Alzheimer's disease; v) history of any serious medical, psychiatric or neurologic disorders; vi) substance abuse; vii) head trauma or loss of consciousness; and viii) any contraindications to MRI.

Laboratory assessments. HbA1c was measured using a high-performance liquid chromatography assay (D-100 system; Bio-Rad Laboratories, Inc.). C-peptide was measured using a chemiluminescent immunometric assay, which employs the Modular Analytics E170 (Roche Diagnostics $\mathrm{GmbH}$ ). Blood glucose, serum creatinine and lipid profiles (total cholesterol and triglycerides) were measured by enzymatic assays (Olympus AU5400 autoanalyzer; Beckman Coulter).

Nerve assessment. Three independently-trained doctors assessed the nerve and mental conditions of the patients in accordance with the pain symptoms and neurological signs: i) The visual analogue scale (VAS) $(12,14)$; ii) the Toronto Clinical Scoring System (TCSS) $(12,14)$; and iii) the Leeds Assessment of Neuropathic Symptoms and Signs $(12,15)$.

MRI acquisition. The whole-blood oxygen level-dependent (BOLD) signals were collected using an Ingenia 3.0T MRI machine (Philips Medical Systems B.V.). The MRI scanning technique was performed as described previously (12). The parameters of T1-weighted imaging were as follows: i) Repetition time (TR)/echo time (TE), 8.2/3.8 msec; ii) field of view (FOV), 240x240 mm; iii) matrix, 240x222; iv) slice thickness, $1 \mathrm{~mm}$; and v) scanning time, $5 \mathrm{~min}$ and $29 \mathrm{sec}$. For subjects with no structural brain abnormalities, a resting-state functional imaging scan was performed. Subjects were required to close their eyes during the scan, and stay awake and quiet without any further movements. The parameters were as follows: i) TR/TE, 2,000/30 msec; ii) FOV, 220x220 mm; iii) matrix, $72 \times 70$, slice thickness, $4 \mathrm{~mm}$; and iv) scanning time, $12 \mathrm{~min}$ and $45 \mathrm{sec}$.

MRI processing. The standard pre-processing steps were performed using the Statistical Parametric Mapping (SPM) version 8 (http://www.fil.ion.ucl.ac.uk/spm/) and the Data Processing Assistant for re-fMRI on the MATLAB R2012b platform (MathWorks) (16).

The initial 10 time-points were removed to eliminate early detection interference. Subsequently, slice timing and head-motion correction were performed. Head movements were calibrated with $2-\mathrm{mm}$ translations and were angled at 2 degrees to eliminate inconsistencies. Subsequently, the image space was normalized in accordance with the Montreal standard head anatomic template and was resampled to a $3 \mathrm{~mm}$ x $3 \mathrm{~mm}$ x $3 \mathrm{~mm}$ size using a unified segmentation algorithm on the T1 image (12). Nuisance signals, including white matter signals and cerebral spinal fluid, were regressed. Detrending and temporal band-pass filtering $(0.01 .0 .08 \mathrm{~Hz})$ were performed. A Gauss kernel function of $4 \mathrm{~mm}$ with full width and half height were used for spatial smoothing.

FC analysis. The thalamus was divided into two subregions (Thalamus_L and Thalamus_R) for selecting the regions of interest (ROIs). Masks of ROIs were obtained using the WFU Pick Atlas 3.0.5 (http://fmri.wfubmc.edu/software/PickAtlas) from the Montreal Neurological Institute, which automatically generated segmented atlas ROI templates.

The mean time course for calculating thalamic ROI was determined by averaging the time course of the voxels within the thalamus (17). Subsequently, the thalamo-cortical FC was calculated. The $\mathrm{z}$ scores were obtained from the correlation coefficients by Fisher's transformation $(18,19)$.

Statistical analysis. To examine inter-group differences in the clinical characteristics, one-way analysis of variance 
Table I. Demographic and clinical characteristics of the participants.

\begin{tabular}{|c|c|c|c|c|}
\hline Item & Group-PDN (n=19) & Group-NDN (n=20) & Group-C $(n=13)$ & P-value \\
\hline Sex (male/female) & $12 / 7$ & $13 / 7$ & $7 / 6$ & $0.800^{\mathrm{a}}$ \\
\hline Age (year) & $53.8 \pm 8.1$ & $54.1 \pm 6.4$ & $53.9 \pm 5.3$ & $0.994^{\mathrm{b}}$ \\
\hline Education (years) & $9.6 \pm 3.4$ & $10.5 \pm 3.7$ & $10.4 \pm 2.9$ & $0.724^{\mathrm{b}}$ \\
\hline Duration of disease (months) & $109.4 \pm 65.5$ & $100.4 \pm 66.9$ & - & $0.671^{\mathrm{c}}$ \\
\hline Fasting blood glucose (mmol/l) & $9.2 \pm 2.9$ & $7.4 \pm 3.5$ & - & $0.083^{c}$ \\
\hline HbA1c $(\%)$ & $8.5 \pm 1.9$ & $8.7 \pm 1.7$ & - & $0.633^{c}$ \\
\hline C-peptide (ng/ml) & $1.6 \pm 0.9$ & $1.2 \pm 0.7$ & - & $0.100^{\mathrm{c}}$ \\
\hline Cholesterol (mmol/l) & $4.9 \pm 1.2$ & $5.1 \pm 1.2$ & - & $0.650^{\mathrm{c}}$ \\
\hline Triacylglycerol (mmol/l) & $2.4 \pm 2.7$ & $2.4 \pm 2.9$ & - & $0.970^{c}$ \\
\hline Creatinine $(\mu \mathrm{mol} / \mathrm{l})$ & $70.0 \pm 19.2$ & $67.9 \pm 30.6$ & - & $0.990^{\mathrm{c}}$ \\
\hline VAS (score) & $6.8 \pm 1.9$ & $0.0 \pm 0.0$ & - & $<0.0001^{\mathrm{c}}$ \\
\hline TCSS (score) & $9.7 \pm 3.4$ & $6.2 \pm 1.6$ & - & $0.0002^{c}$ \\
\hline
\end{tabular}

Values are expressed as the mean \pm standard deviation or $\mathrm{n} .{ }^{\mathrm{a}}$ Chi-square test, ${ }^{\mathrm{b}} \mathrm{ANOVA}$, ${ }^{\mathrm{c}}$ independent-samples t-test. Group-PDN, T2DM with painful neuropathy; Group-NDN, T2DM with non-painful neuropathy; Group-C, Healthy control; HbA1c, glycated hemoglobin; VAS, visual analogue scale; TCSS, Toronto Clinical Scoring System.

A

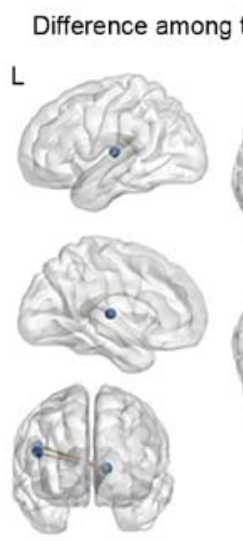

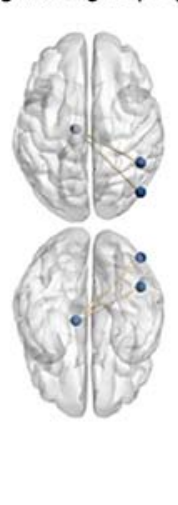

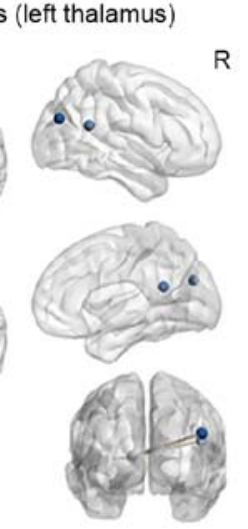

R

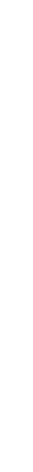

B

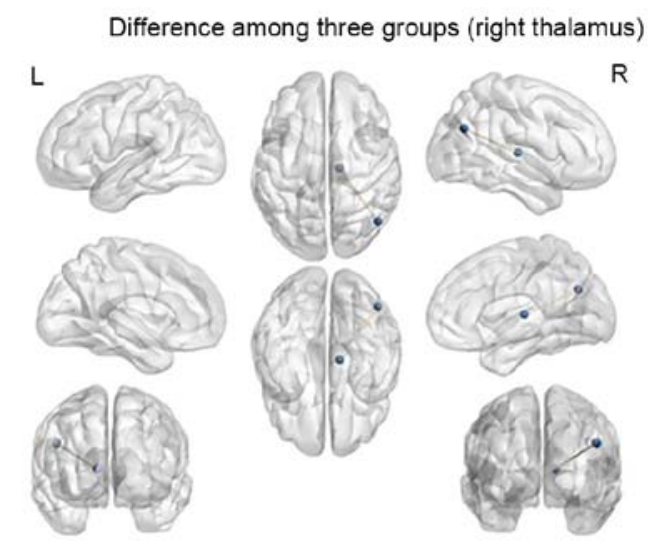

Figure 1. Differences in functional connectivity in the thalamus among the three groups. The blue dots represent brain regions that had abnormal activity among the three groups. (A) Brain regions exhibiting different activity among three groups when considering the left thalamus as the ROI. (B) Brain regions exhibiting different activity among the three groups when considering the right thalamus as the ROI. ROI, region of interest.

(ANOVA), an independent-samples t-test and the $\chi^{2}$ test were applied using SPSS 22.0 software (IBM Corp.).

To compare differences in rs-FC among three groups, ANOVA was performed using the resting-state fMRI data analysis toolkit (REST) software (20). The t-test was conducted to explore differences in rs-FC between of PDN and NDN groups by treating age, sex and years of education as covariates. Multiple comparative corrections were performed using Monte Carlo simulation in conjunction with the REST AlphaSim program. Voxels with cluster sizes $\geq 4$ $\left(108 \mathrm{~mm}^{3}\right)$ and $\mathrm{P}<0.01$ were regarded as significant brain areas, corresponding to the corrected $\mathrm{P}<0.05$.

\section{Results}

Demographic and clinical characteristics. All available cases were collected. Finally, a total of 52 right-handed subjects were recruited for the present study. The patients with DN were categorized into two groups (PDN and NDN). Group PDN was comprised of 12 males and 8 females, whereas group NDN was comprised of 13 males and 7 females. A total of 13 age-, sex- and years of education-matched healthy volunteers ( 7 males and 6 females) were recruited. One patient was excluded from Group-PDN due to head motion $>2 \mathrm{~mm}$ during MRI. The patient characteristics are presented in Table I.

No significant difference in age, sex and years of educationwas present among the three groups. Furthermore, there was no significant difference in the course of disease, fasting blood glucose, HbA1c, C-peptide, total cholesterol, triacylglycerol and creatinine between the PDN and NDN groups (Table I); however, significant differences in nerve conditions as measured by the VAS score and TCSS were detected between the two groups (Table I).

FC differences between groups. Considering the left thalamus as the ROI, the results of the one-way ANOVA revealed 
Table II. ANOVA of differences in thalamic-whole brain functional connectivity among three groups when taking the bilateral thalamus as the region of interest.

A, FC with the left thalamus

\begin{tabular}{lcrrrrr}
\hline & \multicolumn{5}{c}{ MNI coordinate } & \\
\cline { 3 - 5 } Area & Side & $\mathrm{x}$ & $\mathrm{y}$ & $\mathrm{z}$ & $\mathrm{K}$ & F-value \\
\hline Vermis_10 & $\mathrm{L}$ & 0 & -45 & -30 & 45 & 11.64 \\
Parahippocampal & $\mathrm{R}$ & 24 & 3 & -21 & 7 & 9.44 \\
Temporal_Inf & $\mathrm{R}$ & 54 & -39 & -15 & 9 & 13.26 \\
Fusiform & $\mathrm{R}$ & 24 & -72 & -6 & 15 & 12.52 \\
Thalamus & $\mathrm{R}$ & 6 & -9 & 3 & 26 & 10.8 \\
Temporal_Mid & $\mathrm{R}$ & 48 & -48 & 18 & 6 & 11.34 \\
Rolandic_Oper & $\mathrm{L}$ & -36 & -36 & 18 & 10 & 9.76 \\
Occipital_Mid & $\mathrm{R}$ & 45 & -75 & 27 & 10 & 9.49 \\
Cingulum_Mid & $\mathrm{L}$ & 0 & -30 & 36 & 12 & 10.19 \\
\hline
\end{tabular}

$\mathrm{B}, \mathrm{FC}$ with the right thalamus

\begin{tabular}{lcrrrrr}
\hline & \multicolumn{5}{c}{ MNI coordinate } \\
\cline { 3 - 5 } Area & Side & $\mathrm{x}$ & $\mathrm{y}$ & $\mathrm{z}$ & $\mathrm{K}$ & F-value \\
\hline Cerebelum_Crus2 & $\mathrm{R}$ & 39 & -66 & -45 & 7 & 11.7 \\
Temporal_Inf & $\mathrm{L}$ & -39 & -18 & -27 & 6 & 14.55 \\
Parahippocampal & $\mathrm{R}$ & 24 & 3 & -21 & 11 & 9.97 \\
Pallidum & $\mathrm{R}$ & 27 & -9 & -3 & 19 & 11.16 \\
Thalamus & $\mathrm{R}$ & 12 & -12 & 9 & 40 & 15.44 \\
Thalamus & $\mathrm{L}$ & -9 & -12 & 6 & 6 & 9.89 \\
Angular & $\mathrm{R}$ & 45 & -69 & 30 & 19 & 11.58 \\
Cingulum_Post & $\mathrm{L}$ & 0 & -36 & 27 & 8 & 9.57 \\
Parietal_Sup & $\mathrm{L}$ & -15 & -72 & 42 & 6 & 12.39 \\
Parietal_Sup & $\mathrm{L}$ & -24 & -63 & 54 & 12 & 13.05 \\
Parietal_Sup & $\mathrm{L}$ & -21 & -48 & 63 & 7 & 10.53 \\
\hline
\end{tabular}

$\mathrm{x}, \mathrm{y}, \mathrm{z}$ are the coordinates of primary peak locations in the MNI space. The $t$ statistical value of peak voxels indicated significant differences in FC among three groups $(\mathrm{P}<0.05$, corrected by AlphaSim correction); cluster size is the number of voxels. L, left; R, right; FC, functional connectivity; MNI, Montreal Neurological Institute; Inf, inferior; Mid, middle; Oper, operculum; Post, posterior; Sup, superior.

significant differences in FC values among the three groups in the vermis, right parahippocampal gyrus automated anatomical labeling (aal) (21), right inferior temporal gyrus (aal), right fusiform gyrus, right thalamus (aal), right middle temporal gyrus (aal), left rolandic operculum (aal), right middle occipital gyrus (aal), left median cingulate and paracingulate gyri (aal), right angular gyrus (aal) and right middle occipital gyrus (aal) $(\mathrm{P}<0.05$, Alphasim correction; Table II and Fig. 1). Compared with Group NDN, Group PDN exhibited significantly increased FC between the left thalamus and the right angular gyrus, as well as the right middle occipital lobe ( $\mathrm{P}<0.05$, AlphaSim correction; Table III and Fig. 2).
Table III. Analysis of the differences in thalamic-whole brain functional connectivity between the Group-PDN and Group-NDN when taking the bilateral thalamus as the region of interest.

A, FC with the left thalamus

\begin{tabular}{|c|c|c|c|c|c|c|}
\hline \multirow[b]{2}{*}{ Area } & \multirow[b]{2}{*}{ Side } & \multicolumn{3}{|c|}{$\begin{array}{c}\text { MNI } \\
\text { coordinate }\end{array}$} & \multirow[b]{2}{*}{$\mathrm{K}$} & \multirow[b]{2}{*}{ t-value } \\
\hline & & $\mathrm{x}$ & $\mathrm{y}$ & $\mathrm{z}$ & & \\
\hline Angular gyrus & $\mathrm{R}$ & 48 & -48 & 21 & 6 & 4.83 \\
\hline Middle occipital gyrus & $\mathrm{R}$ & 48 & -75 & 27 & 5 & 4.28 \\
\hline \multicolumn{7}{|c|}{$\mathrm{B}, \mathrm{FC}$ with the right thalamus } \\
\hline & & \multicolumn{3}{|c|}{$\begin{array}{c}\text { MNI } \\
\text { coordinate }\end{array}$} & \multirow[b]{2}{*}{$\mathrm{K}$} & \multirow[b]{2}{*}{ t-value } \\
\hline Area & Side & $\mathrm{x}$ & $\mathrm{y}$ & $\mathrm{z}$ & & \\
\hline Angular gyrus & $\mathrm{R}$ & 48 & -66 & 3 & 19 & 4.47 \\
\hline \multicolumn{7}{|c|}{$\begin{array}{l}\mathrm{x}, \mathrm{y}, \mathrm{z} \text { are the coordinates of primary peak locations in the MNI space. } \\
\text { The } t \text { statistical value of peak voxels indicated significant differences } \\
\text { in FC between the groups PDN and NDN (P<0.05, corrected by } \\
\text { AlphaSim correction); cluster size is the number of voxels. R, right; } \\
\text { FC, functional connectivity; MNI, Montreal Neurological Institute; } \\
\text { Group-PDN, T2DM with painful neuropathy; Group-NDN, T2DM } \\
\text { with non-painful neuropathy. }\end{array}$} \\
\hline
\end{tabular}

Considering the right thalamus as the ROI, the results of the one-way ANOVA indicated that significant differences in FC values among three groups were present in the right cerebellum, left inferior temporal gyrus, right parahippocampal gyrus (aal), right lenticular nucleus, pallidum (aal), right thalamus, left thalamus, right angular gyrus (aal), left posterior cingulate gyrus and left superior parietal gyrus (aal) $(\mathrm{P}<0.05$, Alphasim correction; Table II and Fig. 1). Compared with Group NDN, Group PDN exhibited significantly increased FC between the right thalamus and the right angular gyrus $(\mathrm{P}<0.05$ AlphaSim correction; Table III and Fig. 2).

\section{Discussion}

Functional MRI has been widely used to investigate brain dysfunction in patients with psychiatric disorders (22-25). To the best of our knowledge, the present study was the first to indicate enhanced thalamic-whole brain FC in Chinese patients with PDN using thalamic seed-based analysis. The results indicated that Group PDN had increased FC between the bilateral thalami and the right angular gyrus and between the left thalamus and the right occipital lobe, when compared with Group NDN. Furthermore, it was determined that Group PDN had increased FC between the left thalamus and the right angular gyrus, as well as the right occipital gyrus. Group PDN also had an increased FC between the right thalamus and the right angular gyrus. 

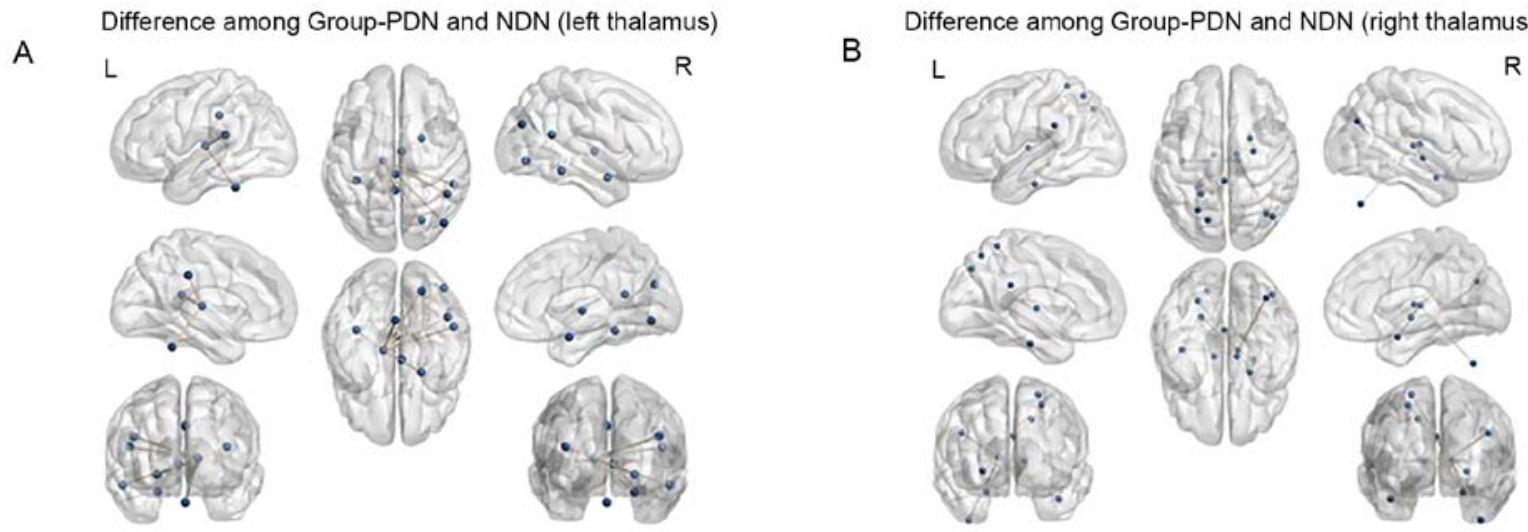

Figure 2. Differences of functional connectivity in the thalamus between Group-PDN and Group-NDN. The blue dots represent brain regions that had significantly increased activity in Group PDN when compared with Group NDN. (A) Brain regions exhibiting different activity between the two groups when considering the left thalamus as the ROI. (B) Brain regions exhibiting different activity between two groups when considering the right thalamus as the ROI. ROI, region of interest; Group-PDN, T2DM with painful neuropathy; Group-NDN, T2DM with non-painful neuropathy.

The thalamus is the central cortical-subcortical connectivity hub and serves as the gateway to the cerebral cortex. The thalamus is considered to have a significant role in the process of pain sensation (26). The thalamus was indicated to be associated with spontaneous and evoked pain in chronic conditions (7). In 2011, it was reported that PDN was associated with increased thalamic vascularity in T1DM (27). To date, there has been a scarcity of research focusing particularly on the thalamus and the role of PDN in T2DM using rs-fMRI. Therefore, the present study may expand the current understanding of the underlying neurobiological mechanisms of these conditions.

The angular gyrus is one of the components within the default mode network (DMN), which was reported to be involved in the process of episodic memory retrieval (28). The angular gyrus is involved in several cognitive domains (i.e., language processing and attention) and serves as an important node of the DMN $(29,30)$. The DMN was indicated to be altered in chronic pain diseases such as fibromyalgia, which is considered to be the prototypical central chronic pain syndrome (31). Another previous study indicated that music was able to reduce pain and increase the amplitude of rs-fMRI BOLD signals in the left angular gyrus in patients with fibromyalgia. The angular gyrus is involved in the top-down regulation of the pain modulatory network by the DMN (32). Furthermore, increased activity in the left angular gyrus following verbally-induced placebo analgesia was observed in patients with chronic pain (33). These results suggested that the angular gyrus may have interactions with brain areas or pathways involved in pain modulation (33).

A previous multivariate analysis of resting FC demonstrated that the angular gyrus had similar connectivity to that in the default mode network (DMN) (28). Another study determined that the angular gyrus were involved in numerous tasks and processes (29). In the present study, it was indicated that in PDN group the bilateral thalamus had increased FC with the right angular gyrus, which was related to the pain modulatory network. Therefore, it was speculated that impaired FC between the thalamus and angular gyrus in PDN may be related to the pain modulatory network within the DMN.
The present study also indicated that the thalamus had increased FC with the right middle occipital gyrus. The thalamus is responsible for interpreting visual information and forming conscious perception (34). Changes in the thickness of the middle occipital gyrus associated were determined in blind patients (35). Furthermore, local synchronicity and abnormal occipital lobe function were reported in patients with hemifacial spasm (36). Patients with classical trigeminal neuralgia (CTN) had symptoms characterized by orbital pain, decreased corneal reflex and decreased vision (34). Individuals with chronic post-hysterectomy pain had decreased FC between the left sensorimotor insula and the right angular and middle occipital gyri (MOG), as well as between the left chemosensory insula, the bilateral angular gyri and the MOG following pain stimulation (37).

Furthermore, increased regional homogeneity (ReHo) values in the right middle occipital gyrus were reported in patients with CTN (34). Patients with drug-induced headaches exhibited an increased volume of the bilateral middle occipital gyrus (38). Furthermore, patients with pain-related conditions had altered neuronal activity or structural functions in the middle occipital gyrus $(38,39)$. These results suggested that the middle occipital gyrus may participate in the processes leading to the perception of pain in patients with diabetic polyneuropathy. The abnormal FC between the thalamus and other brain regions were indicated to be associated with PDN.

The present study has several limitations. First, the sample size was small. Furthermore, no associations between the altered brain regions and clinical characteristics were explored in the present study. Further studies with a larger sample size are required to understand the mechanisms of T2DM with PDN. Finally, it was not possible to exclude the potential influences of medication, such as oral antidiabetic drugs, in the present study.

In conclusion, the present study revealed enhanced FC between the bilateral thalamic-angular gyrus and the left thalamic-right MOG in patients with T2DM and PDN. The increased thalamic-whole brain FC may be involved in PDN. Furthermore, the abnormal thalamic-angular gyrus FC may be related to the DMN. These results may be helpful for 
understanding the central pathophysiological mechanisms of PDN in patients with T2DM.

\section{Acknowledgements}

Not applicable.

\section{Funding}

The present study was supported by grants from the Nanjing Science and Technology Development Project (grant no. 201605027) and the Natural Science Foundation of Jiangsu Province (grant no. BK20170136).

\section{Availability of data and materials}

The datasets used and/or analyzed during the current study are available from the corresponding author on reasonable request.

\section{Authors' contributions}

$\mathrm{XL}, \mathrm{XX}$ and CM acquired and interpreted the patient data, constructed the figures and wrote the manuscript. PZ, QZ, LJ, YY, JM, LY and KL acquired and analyzed the patient data, and revised the manuscript. JW and ZY designed the study and revised the manuscript. JW and ZY confirm the authenticity of all the raw data. All authors have read and approved the final manuscript.

\section{Ethics approval and consent to participate}

The protocol was approved by the Institutional Review Board of Nanjing First Hospital (Nanjing, China). All procedures were in accordance with the 1964 Helsinki Declaration and its later amendments or comparable ethical standards. Written informed consent was obtained from all participants.

\section{Patient consent for publication}

Not applicable.

\section{Competing interests}

The authors declare that they have no competing interests.

\section{References}

1. Feldman EL, Nave KA, Jensen TS and Bennett DLH: New horizons in diabetic neuropathy: Mechanisms, bioenergetics, and pain. Neuron 93: 1296-1313, 2017.

2. Gaskin DJ and Richard P: The economic costs of pain in the United States. J Pain 13: 715-724, 2012.

3. Fayaz A, Croft P, Langford RM, Donaldson LJ and Jones GT: Prevalence of chronic pain in the UK: A systematic review and meta-analysis of population studies. BMJ Open 6: e010364, 2016.

4. Dermanovic Dobrota V, Hrabac P, Skegro D, Smiljanic R, Dobrota S, Prkacin I, Brkljacic N, Peros K, Tomic M, Lukinovic-Skudar V and Basic Kes V: The impact of neuropathic pain and other comorbidities on the quality of life in patients with diabetes. Health Qual Life Outcomes 12: 171, 2014.

5. Tesfaye S, Selvarajah D, Gandhi R, Greig M, Shillo P, Fang F and Wilkinson ID: Diabetic peripheral neuropathy may not be as its name suggests: Evidence from magnetic resonance imaging. Pain 157 (Suppl 1): S72-S80, 2016.
6. Modha DS and Singh R: Network architecture of the long-distance pathways in the macaque brain. Proc Natl Acad Sci USA 107: 13485-13490, 2010.

7. Yen CT and Lu PL: Thalamus and pain. Acta Anaesthesiol Taiwan 51: 73-80, 2013.

8. Selvarajah D, Wilkinson ID, Maxwell M, Davies J, Sankar A, Boland E, Gandhi R, Tracey I and Tesfaye S: Magnetic resonance neuroimaging study of brain structural differences in diabetic peripheral neuropathy. Diabetes Care 37: 1681-1688, 2014.

9. Biswal B, Yetkin FZ, Haughton VM and Hyde JS: Functional connectivity in the motor cortex of resting human brain using echo-planar MRI. Magn Reson Med 34: 537-541, 1995.

10. Zuo XN, Kelly C, Di Martino A, Mennes M, Margulies DS Bangaru S, Grzadzinski R, Evans AC, Zang YF, Castellanos FX and Milham MP: Growing together and growing apart: Regional and sex differences in the lifespan developmental trajectories of functional homotopy. J Neurosci 30: 15034-15043, 2010.

11. Gorges M, Muller HP, Lule D, Ludolph AC, Pinkhardt EH and Kassubek J: Functional connectivity within the default mode network is associated with saccadic accuracy in Parkinson's disease: A resting-state FMRI and videooculographic study. Brain Connect 3: 265-272, 2013.

12. Zhang Q, Zhang P, Yan R, Xu X, Mao C, Liu X, Li F, Ma J, Ye L, Yao $\mathrm{Z}$ and $\mathrm{Wu} \mathrm{J}$ : A single-blinded trial using resting-state functional magnetic resonance imaging of brain activity in patients with type 2 diabetes and painful neuropathy. Diabetes Ther 10: 135-147, 2019.

13. Gabir MM, Hanson RL, Dabelea D, Imperatore G, Roumain J, Bennett PH and Knowler WC: The 1997 American Diabetes Association and 1999 World Health Organization criteria for hyperglycemia in the diagnosis and prediction of diabetes. Diabetes Care 23: 1108-1112, 2000.

14. Essmat A and Hussein MS: Green tea extract for mild-to-moderate diabetic peripheral neuropathy A randomized controlled trial. Complement Ther Clin Pract 43: 101317, 2021.

15. Garoushi S, Johnson MI and Tashani OA: Translation and cultural adaptation of the Leeds Assessment of Neuropathic Symptoms and Signs (LANSS) pain scale into Arabic for use with patients with diabetes in Libya. Libyan J Med 12: 1384288, 2017.

16. Chao-Gan Y and Yu-Feng Z: DPARSF: A MATLAB toolbox for 'Pipeline' data analysis of resting-state fMRI. Front Syst Neurosci 4: 13, 2010.

17. Salvador R, Suckling J, Schwarzbauer C and Bullmore E: Undirected graphs of frequency-dependent functional connectivity in whole brain networks. Philos Trans R Soc Lond B Biol Sci 360: 937-946, 2005.

18. Liu Y, Yu C, Liang M, Li J, Tian L, Zhou Y, Qin W, Li K and Jiang T: Whole brain functional connectivity in the early blind. Brain 130: 2085-2096, 2007.

19. Hale JR, White TP, Mayhew SD, Wilson RS, Rollings DT, Khalsa S, Arvanitis TN and Bagshaw AP: Altered thalamocortical and intra-thalamic functional connectivity during light sleep compared with wake. Neuroimage 125: 657-667, 2016.

20. Song XW, Dong ZY, Long XY, Li SF, Zuo XN, Zhu CZ, He Y, Yan CG and Zang YF: REST: A toolkit for resting-state functional magnetic resonance imaging data processing. PLoS One 6: e25031, 2011.

21. Tzourio-Mazoyer N, Landeau B, Papathanassiou D, Crivello F, Etard O, Delcroix N, Mazoyer B and Joliot M: Automated anatomical labeling of activations in SPM using a macroscopic anatomical parcellation of the MNI MRI single-subject brain. Neuroimage 15: 273-289, 2002.

22. Zhu X, He Z, Luo C, Qiu X, He S, Peng A, Zhang L and Chen L: Altered spontaneous brain activity in MRI-negative refractory temporal lobe epilepsy patients with major depressive disorder: A resting-state fMRI study. J Neurol Sci 386: 29-35, 2018.

23. Tose K, Yoshihara Y and Takahashi H: FMRI neurofeedback and its application to psychiatric disorders. Brain Nerve 70: 1209-1216, 2018

24. Tadayonnejad R, Deshpande R, Ajilore O, Moody T, Morfini F, Ly R, O'Neill J and Feusner JD: Pregenual anterior cingulate dysfunction associated with depression in OCD: An integrated multimodal fMRI/(1)H MRS study. Neuropsychopharmacology 43: 1146-1155, 2018.

25. Shen Z, Jiang L, Yang S, Ye J, Dai N, Liu X, Li N, Lu J, Liu F, Lu Y, et al: Identify changes of brain regional homogeneity in early and later adult onset patients with first-episode depression using resting-state fMRI. PLoS One 12: e0184712, 2017.

26. Head H and Holmes G: Sensory disturbances from cerebral lesions. Brain 34: 102-254, 1911. 
27. Selvarajah D, Wilkinson ID, Gandhi R, Griffiths PD and Tesfaye S: Microvascular perfusion abnormalities of the Thalamus in painful but not painless diabetic polyneuropathy: A clue to the pathogenesis of pain in type 1 diabetes. Diabetes Care 34: 718-720, 2011.

28. Bellana B, Liu Z, Anderson JAE, Moscovitch M and Grady CL: Laterality effects in functional connectivity of the angular gyrus during rest and episodic retrieval. Neuropsychologia 80: 24-34, 2016.

29. Seghier ML: The angular gyrus: Multiple functions and multiple subdivisions. Neuroscientist 19: 43-61, 2013.

30. Greicius MD, Supekar K, Menon V and Dougherty RF: Resting-state functional connectivity reflects structural connectivity in the default mode network. Cereb Cortex 19: 72-78, 2009.

31. Napadow V, LaCount L, Park K, As-Sanie S, Clauw DJ and Harris RE: Intrinsic brain connectivity in fibromyalgia is associated with chronic pain intensity. Arthritis Rheum 62: 2545-2555, 2010.

32. Garza-Villarreal EA, Jiang Z, Vuust P, Alcauter S, Vase L, Pasaye EH, Cavazos-Rodriguez R, Brattico E, Jensen TS and Barrios FA: Music reduces pain and increases resting state fMRI BOLD signal amplitude in the left angular gyrus in fibromyalgia patients. Front Psychol 6: 1051, 2015.

33. Craggs JG, Price DD and Robinson ME: Enhancing the placebo response: Functional magnetic resonance imaging evidence of memory and semantic processing in placebo analgesia. J Pain 15: 435-446, 2014.

34. Xiang CQ, Liu WF, Xu QH, Su T, Yong-Qiang S, Min YL, Yuan Q, Zhu PW, Liu KC, Jiang N, et al: Altered spontaneous brain activity in classical trigeminal neuralgia as determined by changes in regional homogeneity: A resting-state functional MRI study. Pain Pract 19: 397-406, 2018.
35. Anurova I, Renier LA, De Volder AG, Carlson S and Rauschecker JP: Relationship between cortical thickness and functional activation in the early blind. Cereb Cortex 25: 2035-2048, 2015.

36. Tu Y, Wei Y, Sun K, Zhao W and Yu B: Altered spontaneous brain activity in patients with hemifacial spasm: A resting-state functional MRI study. PLoS One 10: e0116849, 2015.

37. Ching YY, Wang C, Tay T, Loke YM, Tang PH, Sng BL and Zhou J: Altered sensory insular connectivity in chronic postsurgical pain patients. Front Hum Neurosci 12: 483, 2018.

38. Chen X, Chen Z, Dong Z, Liu M and Yu S: Morphometric changes over the whole brain in caffeine-containing combination-analgesic-overuse headache. Mol Pain 14: $1744806918778641,2018$.

39. Chen Y, Meng Z, Zhang Z, Zhu Y, Gao R, Cao X, Tan L, Wang Z, Zhang $\mathrm{H}$, Li Y and Fan Q: The right thalamic glutamate level correlates with functional connectivity with right dorsal anterior cingulate cortex/middle occipital gyrus in unmedicated obsessive-compulsive disorder: A combined fMRI and ${ }^{1} \mathrm{H}-\mathrm{MRS}$ study. Aust N Z J Psychiatry 53: 207-218, 2019.

(i) (9) This work is licensed under a Creative Commons Attribution-NonCommercial-NoDerivatives 4.0 International (CC BY-NC-ND 4.0) License. 